\title{
Neutron scattering and extra-short-range interactions
}

\author{
V. V. Nesvizhevsky ${ }^{*}$ \\ Institute Laue Langevin, 6 rue Jules Horowitz, F-38042, Grenoble, France \\ G. Pigno用 and K. V. Protasov团 \\ Laboratoire de Physique Subatomique et de Cosmologie, \\ UJF - CNRS/IN2P3 - INPG, 53 Av. des Martyrs, Grenoble, France
}

(Dated: October 23, 2018)

\begin{abstract}
The available data on neutron scattering were analysed to constrain a hypothetical new shortrange interaction. We show that these constraints are several orders of magnitude better than those usually cited in the range between $1 \mathrm{pm}$ and $5 \mathrm{~nm}$. This distance range occupies an intermediate space between collider searches for strongly coupled heavy bosons and searches for new weak macroscopic forces. We emphasise the reliability of the neutron contraints in so far as they provide several independent strategies. We have identified the most promising way to improve them.

PACS numbers: 03.75.Dg, 03.75.Be, 13.40.Gp
\end{abstract}

\section{INTRODUCTION}

The existence of other forces in nature, mediated by new bosons, has been extensively discussed in the literature, given their possibility in many of the extensions to the standard model of particle physics [1]. New bosons for example are predicted by most of the Grand Unified Theories embedding the standard model, with a coupling constant of $\approx 10^{-1}$. These strongly coupled bosons would have to be heavier than $\approx 1 \mathrm{TeV}$ if they were not to conflict with present observations; heavier bosons will be searched for at the Large Hadron Collider. Lighter bosons could however have remained unnoticed, provided they interact weakly with matter. Such bosons would mediate a finite range force between two fermions:

$$
V(r)=Q_{1} Q_{2} \frac{g^{2}}{4 \pi} \frac{\hbar c}{r} e^{-r / \lambda}
$$

where $g$ is the coupling constant, $Q_{1}$ and $Q_{2}$ the charges of the fermions under the new interaction, and the range of this Yukawa-like potential $\lambda=\frac{\hbar}{M c}$ is inversely proportional to the boson mass $M$. In the following we consider the interactions of neutrons with nuclei of atomic number $A$ : the charge of the atom under the new interaction is equal $Q_{1}=A$; the neutron charge is equal unity $Q_{2}=1$. A new boson could even be massless, as has been suggested by Lee and Yang 2] well before the birth of the standard model, to explain the conservation of the baryon number. This additional massless boson would mediate a new infinite-range force, and could be seen in searches for violation of the equivalence principle. The presence of very light bosons $(M \ll 1 \mathrm{eV}$ ) would be shown by deviations from the gravitational inverse square law. Gravity has been probed down to distances of $0.1 \mathrm{~mm}[3$; new

\footnotetext{
*Electronic address: nesvizhevsky@ill.eu

${ }^{\dagger}$ Electronic address: pignol@lpsc.in2p3.fr

${ }^{\ddagger}$ Electronic address: protasov@lpsc.in2p3.fr
}

bosons lighter than $2 \times 10^{-3} \mathrm{eV}$ must thus have a coupling constant lower than the gravity strength between nucleons, $g^{2}<10^{-37}$.

Theories with extra large spatial dimensions [4, 5, 6, 7, 8, 9] provide strong motivation to search for such forces. If a boson is allowed to travel in large extra-dimensions, with a strong coupling constant in the bulk, it behaves in our 4D world as a very weakly coupled new boson, the coupling being diluted in the extra-dimensions. The light dark matter hypothesis also argues in favour of the existence of new short range interactions [10].

While measurements of the Casimir or Van der Waals forces (for a review, see e.g. [11]) give the best constraints in the nanometer range $(10 \mathrm{~nm}<\lambda<1 \mu \mathrm{m})$, and antiprotonic atoms constrain the domain below 1 pm [12, 13], it has been suggested that experiments with neutrons could be competitive in the intermediate range [13, 14, 15, 16, 17, 18]. Neutrons could also probe spindependent interactions in a wider distance range [19], or spin-independent interactions in the range of several micrometers [18, 20].

In this article we give the quantitative constraints on the parameters of the additional interaction, $\lambda$ and $g$, from the existing data on neutrons scattering at nuclei. In section[I] we analyse the influence of a new short-range interaction on the scattering of neutrons at nuclei. In section III, we use the fact that the nuclear radius, as well as the scattering lengths, are expected to be proportional to $A^{1 / 3}$, where $A$ is the number of nucleons, whilst the contribution of an additional interaction would result in an additional linear term in the mass dependence of the scattering lengths. In section [V] and $[\mathrm{V}$, we use the different sensitivities of different types of neutron scattering experiments to extra interactions (forward and backward scattering) in order to constrain them. In section VI we propose a way to improve these constraints [18]. 


\section{SLOW NEUTRON / NUCLEI INTERACTION WITH EXTRA-SHORT-RANGE INTERACTIONS}

The scattering of slow neutrons on atoms is described by the scattering amplitude $f(\mathbf{q})$; this can be represented by a sum of a few terms [21]:

$$
f(\mathbf{q})=f_{\text {nucl }}(\mathbf{q})+f_{n e}(\mathbf{q})+f_{V}(\mathbf{q})
$$

The first and the most important term represents the scattering due to the nuclear neutron-nucleus interaction. At low energies discussed in this article, it is isotropic and energy-independant, because the nuclear radius is much smaller than the wavelegth of slow neutrons:

$$
f_{\text {nucl }}(\mathbf{q})=-b .
$$

The coherent scattering lenght $b$ is the fundamental parameter describing the interaction of slow neutrons with a nucleus [22].

The second term is the amplitude of so-called electronneutron scattering due to the interaction of the neutron charge distribution with the nucleus charge and the electron cloud. This amplitude can be written as

$$
f_{n e}(\mathbf{q})=-b_{n e}(Z-f(Z, \mathbf{q})),
$$

where $f(Z, \mathbf{q})$ is the atomic form-factor measured in the X-rays experiments and $b_{n e}$ is a constant called the electron-neutron scattering length, which is directly related to the neutron charge radius [21] and to the neutron electromagnetic form-factor $G_{E}\left(\mathbf{q}^{2}\right)$ by

$$
b_{n e}=-\left.\frac{2}{a_{0}} \frac{m}{m_{e}} \frac{d G_{E}\left(\mathbf{q}^{2}\right)}{d \mathbf{q}^{2}}\right|_{\mathbf{q}^{2}=0},
$$

$m$ and $m_{e}$ being the neutron and electron masses, $a_{0}$ the Bohr radius. This contribution to the total scattering amplitude is as small as a per cent for heavy nuclei.

In the presence of a new interaction (11), the scattering for a center of mass momentum $\hbar k$ due to the extra interaction, within the Born approximation, is given by

$$
f_{V}(\theta)=-A \frac{g^{2}}{4 \pi} \hbar c \frac{2 m \lambda^{2} / \hbar^{2}}{1+(q \lambda)^{2}}
$$

where $q=2 k \sin (\theta / 2), \theta$ is the scattering angle.

Any other possible contributions to the scattering amplitude $f(\mathbf{q})$, due to non zero nuclear radius, nucleon polarizability, etc. are very small in the energy range discussed here 21] and have therefore been omitted in (21).

The nuclear scattering lengths are measured for almost all stable nuclei, using a variety of methods. A review of the different methods and a complete table of the measured scattering lengths can be found in [23]. We can distinguish two classes of method, with different sensitivities to a new interaction.

The first class - including the interference method, the total reflection method, the gravity refractometer method - measures the forward scattering amplitude $f(\mathbf{q}=0)$. These methods actually measure the mean optical potential of a given material, called the Fermi potential, due to the coherent scattering of neutrons at many nuclei. The Fermi potential is related to the forward scattering amplitude.

In the presence of the new force, the measured scattering lenght can be separated into a nuclear and an additional term [29]:

$$
b_{\mathrm{opt}}=-f(\mathbf{q}=0)=b+A \frac{m c^{2}}{2 \pi \hbar c} g^{2} \lambda^{2}
$$

The second class of method - including the Bragg diffraction method and the transmission method - uses nonzero transferred momentum. In the Bragg diffraction method, the scattering amplitude for a momentum transfer of $q_{\mathrm{BD}}=10 \mathrm{~nm}^{-1}$ is measured. One actually extracts, besides the nuclear term, an extra contribution according to (6)

$$
b_{\mathrm{BD}}=b+A \frac{m c^{2}}{2 \pi \hbar c} g^{2} \frac{\lambda^{2}}{1+\left(q_{\mathrm{BD}} \lambda\right)^{2}}
$$

In the case of the transmission method, the total crosssection is measured. Generally, neutrons with energies of about $1 \mathrm{eV}$ are used; they are much faster than slow neutrons, and no coherent scattering can be observed. An additional interaction would manifest itself by an energy dependance of the extracted scattering length

$$
b_{\mathrm{TR}}\left(k^{2}\right)=\sqrt{\frac{\sigma_{\mathrm{tot}}}{4 \pi}}=b+A \frac{m c^{2}}{2 \pi \hbar c} g^{2} \lambda^{2} \frac{\ln \left(1+4(k \lambda)^{2}\right)}{4(k \lambda)^{2}}
$$

Finally, we should also mention the very popular Christiansen filter technique; this measures relative scattering lengths, so we do not consider this data.

\section{RANDOM POTENTIAL NUCLEAR MODEL}

A simple and robust limit on the additional Yukawa forces can be easily obtained by neglecting the small term due to the neutron-electron scattering and by studing the general $A$-dependence of the scattering amplitude. In the domain of $\lambda \leq 1 / q_{\mathrm{BD}}$, the optical and Bragg diffraction methods are sensitive to the same amplitude

$$
b_{\text {Meas }}=-f(\mathbf{q}=0)=b+A \frac{m c^{2}}{2 \pi \hbar c} g^{2} \lambda^{2}
$$

as clear from (77) and (8). The presence of additional forces would be apparent from the linear increase of the measured scattering length as a function of $A$. This dependence is not observed in the experimental data presented in fig. 1. Strong variations with $A$ of the measured scattering length are mostly due to the shell effects in neutron-nucleus nuclear amplitude $b$.

To establish a quantitative upper limit on this additional term, the $A$ dependence of the nuclear scattering 


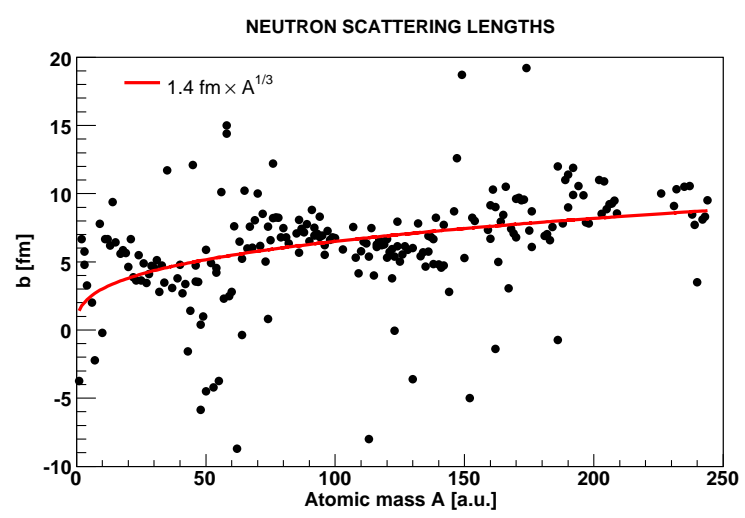

FIG. 1: Measured scattering lengths as a function of nucleus atomic number.

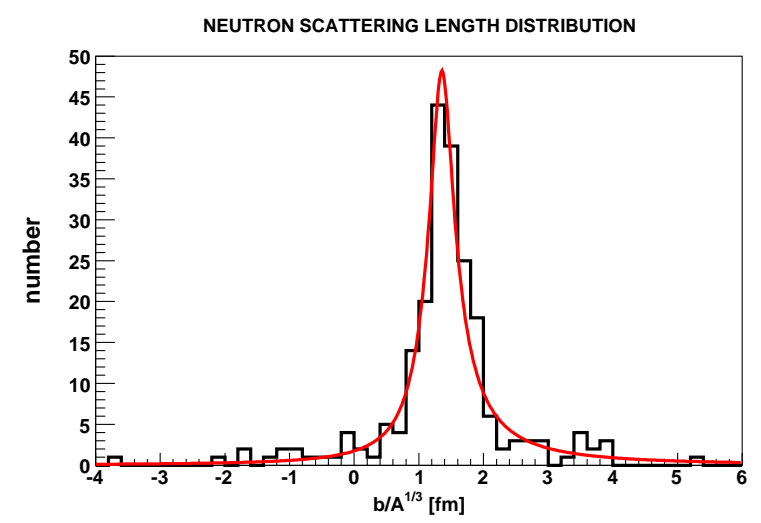

FIG. 2: This histogram shows the distribution of measured scattering lengths normalized to the radius of the nuclei. The curve corresponds to the random potential model.

length $b=b(A)$ needs to be taken into account. The complete $a b$ initio calculation of these shell effects for $b$ is particularly complicated and has never been reported in the literature. Fortunately, there exists a very simple and elegant semi-phenomenological approach that describes these variations [25]. It assumes that a nucleus can be presented as an attractive "square well" potential, with radius $R A^{1 / 3}$ and depth $V_{0}$ for slow neutrons. The scattering length would then be equal to

$$
b(A)=R A^{1 / 3}\left(1-\frac{\tan (X)}{X}\right)
$$

where $X=\frac{R A^{1 / 3}}{\hbar} \sqrt{2 m V_{0}}$ is supposed to be a random variable distributed uniformly over the range $[\pi / 2,5 \pi / 2]$; the lower value corresponds to the appearance of a bound state and the upper limit is set sufficiently large not to influence the results of the present analysis; more details can be found in 25].

Since 1970 (25]), the number of nuclei with measured scattering lengths has doubled and the level of precision
MAXIMUM LIKELIHOOD ANALYSIS

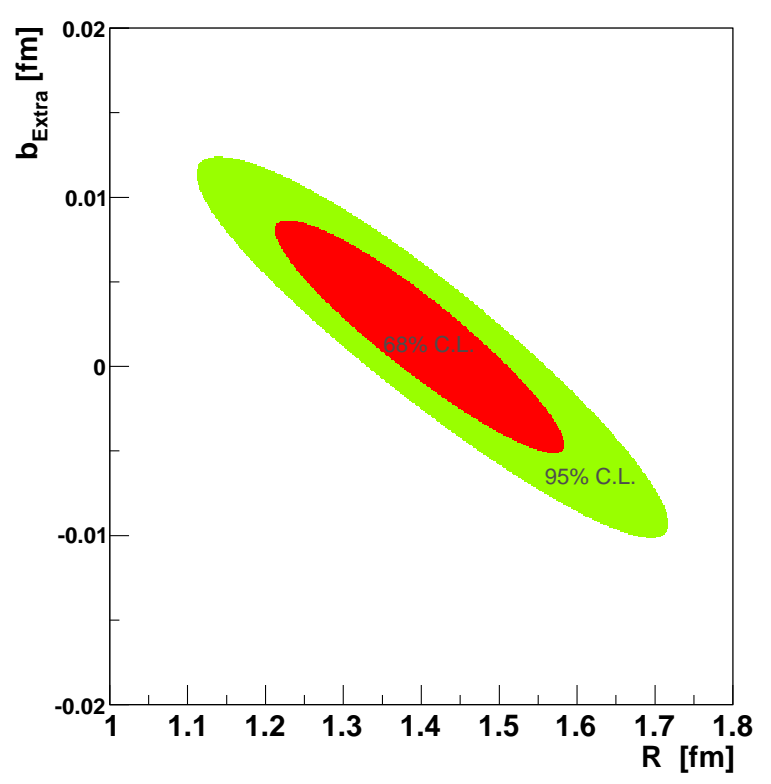

FIG. 3: Maximum likelihood analysis of the two parameters $R$ and $b_{\text {Extra }}$ of (12).

has been improved. We include here the new interaction analysis.

Without any additional interaction, this analysis provides the results presented in fig. 2. One may conclude that the model describes well the distribution of all experimental data well; the value of the only free parameter in this model is estimated to be $R=1.44 \pm 0.05 \mathrm{fm}$ at the $68 \%$ C.L. The likelihood function at its maximum satisfies $\ln (L)=-254$ for 216 degrees of freedom.

With a short-range new interaction included in the analysis we have to consider the random variable

$$
b_{\text {Meas }}=R A^{1 / 3}\left(1-\frac{\tan (X)}{X}\right)+b_{\text {Extra }} A
$$

where the effect of the extra interaction is the slope $b_{\text {Extra }}=\frac{m c^{2}}{2 \pi \hbar c} g^{2} \lambda^{2}$ of the linear term. The estimation of the two parameters $R$ and $b_{\text {Extra }}$ from the experimental data, again using the maximum likelihood method, is presented in fig. 3. The linear term is compatible with zero, as expected. We thus obtain a quantitative constraint for the coupling $g(\lambda)$ :

$$
g^{2} \lambda^{2} \leq 0.016 \mathrm{fm}^{2} \quad \text { at } 95 \% \text { C.L. }
$$

This result is presented in fig. 7 for the distance range of interest, $10^{-12}-10^{-10} \mathrm{~m}$. 


\section{CONSTRAINT FROM COMPARAISON OF FORWARD AND BACKWARD SCATTERING OF NEUTRONS}

Another way to constrain on aditional Yukawa forces consists in comparing the scattering lengths measured by different methods.

As explained above, the scattering lengths measured using the Bragg diffraction method $b_{\mathrm{BD}}$ and the interference method $b_{\text {opt }}$ do not show the same sensitivity to a new short-range interaction. According to (7) and (8), the ratio of the two values should deviate from unity in the presence of an additional interaction

$$
\frac{b_{\mathrm{opt}}}{b_{\mathrm{BD}}} \approx 1+\frac{A}{b} \frac{m c^{2}}{2 \pi \hbar c} g^{2} \lambda^{2} \frac{(q \lambda)^{2}}{1+(q \lambda)^{2}}
$$

We found a set of 13 nuclei for which both measurements exist.

The different measurements quoted in the litterature are sometimes incompatible, even for the same measurement method, because of additional systematics not included in the quoted errors. To compensate for this we estimated a methodological error $\sigma$ for each method. By selecting all the nuclei for which several measurements with a given method are available, we obtained the values $b_{A, i} \pm \Delta b_{A, i}$, where $A$ is the nucleus index and $1 \leqslant i \leqslant n_{A}$ lists the experiments using the given method. The methodological error is then calculated so that:

$$
\sum_{A, i} \frac{\left(b_{A, i}-\bar{b}_{A}\right)^{2}}{\sigma_{A, i}^{2}+\sigma^{2}}=\sum_{A} n_{A} .
$$

That is, we force the $\chi^{2}$ deviation from the weighted average $\bar{b}_{A}$ to be equal to the number of degrees of freedom $\sum_{A} n_{A}$. For the Bragg diffraction method, we have 72 degrees of freedom, and a methodological error of 0.16 fm; this can be compared to the average of the publised errors of $0.23 \mathrm{fm}$. For the interference method, we have 22 degrees of freedom; we found a methodological error of $0.05 \mathrm{fm}$, while the average of published errors is $0.05 \mathrm{fm}$. Table $\llbracket$ summarizes.

With the enlarged errors, a $\chi^{2}$ fit of eq. (14) using the 13 set of data for $b_{\mathrm{I}}$ and $b_{\mathrm{BD}}$, we obtain the constraint:

$$
g^{2} \lambda^{2} \frac{(q \lambda)^{2}}{1+(q \lambda)^{2}} \leq 0.0013 \mathrm{fm}^{2} \quad \text { at } 95 \% \text { C.L. }
$$

corresponding to the bold limit in fig. 7

\section{ELECTROMAGNETIC EFFECTS}

Up to now, the amplitude due to a new additional interaction $f_{V}(\mathbf{q})$ has been compared to the nuclear one $f_{\text {nucl }}(\mathbf{q})$ (see (2)). One could improve the limit obtained by comparing the amplitude due to a new additional interaction to a smaller amplitude due to an electromagnetic interaction $\left(f_{n e}(\mathbf{q})\right)$. This idea was first proposed in ref. [14].
TABLE I: Existing data on neutron scattering lengths. Method Bragg Interference Diffraction

\begin{tabular}{lcc}
\hline Number of measurements & 141 & 41 \\
Number of measured nuclei & 98 & 28 \\
Mean relative accuracy & $3 \%$ & $0.4 \%$ \\
Methodological error [fm] & 0.16 & 0.05
\end{tabular}

To achieve this, three independent measurements are required (roughly speaking, to determine independently the three contributions to (2) ).

One could repeat the previous analysis using measurements of the total cross-section instead of the Bragg diffraction. As can be seen from (9), the scattering length extracted at energies of $\approx 1 \mathrm{eV}\left(k=200 \mathrm{~nm}^{-1}=\right.$ $1 / 5 \mathrm{pm})$ contains an extra force contribution that is different from that in optical methods. In particular, if the range of a new interaction is larger than $1 \mathrm{pm}$, the scattering length extracted from the total cross-section at $1 \mathrm{eV}$ can be considered free of any extra contribution. The sensitivity is so high, however, that the residual electromagnetic effects due to the neutron square charge radius can mimick an extra-force contribution in the quantity $b(1 \mathrm{eV})-b_{\mathrm{opt}}$, as this contribution is energydependent and proportional to the charge number of the atoms. This problem is known as the determination of the neutron-electron scattering length $b_{n e}$. The extracted difference $b(1 \mathrm{eV})-b_{\text {opt }}$ therefore contains the two contributions:

$b(1 \mathrm{eV})-b(0)=Z b_{n e}-A \frac{m c^{2}}{2 \pi \hbar c} g^{2} \lambda^{2}\left(1-\frac{\ln \left(1+4\left(\frac{\lambda}{5 \mathrm{pm}}\right)^{2}\right)}{4\left(\frac{\lambda}{5 \mathrm{pm}}\right)^{2}}\right)$

Unfortunately, there is very clear disagreement between the two groups of values for $b_{n e}^{\exp }=\frac{b(1 \mathrm{eV})-b(0)}{Z}$ known as the Garching-Argonne and Dubna values [26]

$$
\begin{aligned}
& b_{n e}^{\exp }=(-1.31 \pm 0.03) \times 10^{-3} \mathrm{fm} \text { [Gartching-Argonne] } \\
& \left.b_{n e}^{\exp }=(-1.59 \pm 0.04) \times 10^{-3} \mathrm{fm} \text { [Dubna }\right]
\end{aligned}
$$

The discrepancy is much greater than the quoted uncertainties of the experiments and there evidently an unaccounted for systematic error in at least one of the experiments.

In order to overcome this difficulty we could determine $b_{n e}$ from the experimental data on the neutron form factor (5). The simplest way to do this consists in using a commonly accepted general parametrization of the neutron form factor [27]:

$$
G_{E}\left(\mathbf{q}^{2}\right)=-a \mu_{n} \frac{\tau}{1+b \tau} G_{D},
$$

where $\mu_{n}=-1.91 \mu_{B}$ is the neutron anomalous magnetic moment, $\tau=\mathbf{q}^{2} / 4 m^{2}$ and

$$
G_{D}\left(\mathbf{q}^{2}\right)=\frac{1}{\left(1+\mathbf{q}^{2} / 0.71(\mathrm{GeV} / \mathrm{c})^{2}\right)^{2}}
$$




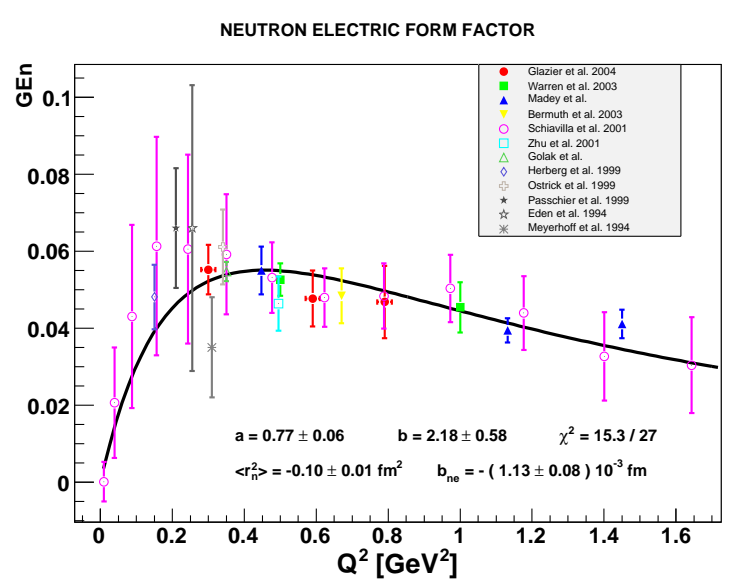

FIG. 4: The neutron form factor $G_{E}\left(\mathbf{q}^{2}\right)$ as a function of the momentum transferred $\mathbf{q}^{2}$. The experimental data are taken from [28]; the solid curve is a two parameter fit using formula (19).

is so-called dipole form factor ; $a$ and $b$ being fitting parameters.

A fit of an existing set of the neutron form factor experimental data [28] yields the following values for the parameters:

$$
\begin{aligned}
& a=(0.77 \pm 0.06) \\
& b=(2.18 \pm 0.58)
\end{aligned}
$$

with $\chi^{2} / \mathrm{NDF}=15.3 / 27$. The results of the fit are presented in fig. [4.

Let us note that the momentum transferred $\mathbf{q}^{2}$ in these experiments is very large and a contribution from the term $f_{V}(\mathbf{q})$ is negligible. The $b_{n e}$ determined in this way is

$$
b_{n e}=(-1.13 \pm 0.08) \times 10^{-3} \mathrm{fm} .
$$

It does not agree with the value of $b_{n e}$, obtained in Dubna experiments.

Our principal conclusion consists in the observation of (underestimated) systematical uncertainties in the presented experiments. Therefore a single experiment/method can not be used for any reliable constraint. A conservative estimate of the precision of the $b_{n e}$ value could be obtained from analysing the discrepancies in the results obtained by different methods; it is equal to $\Delta b_{n e} \leqslant 6 \times 10^{-4} \mathrm{fm}$. The corresponding contraint at the $2 \sigma$ level

$$
\frac{m c^{2}}{2 \pi \hbar c} g^{2} \lambda^{2}\left(1-\frac{\ln \left(1+4\left(\frac{\lambda}{5 \mathrm{pm}}\right)^{2}\right)}{4\left(\frac{\lambda}{5 \mathrm{pm}}\right)^{2}}\right) \leqslant \Delta b_{n e}
$$

is represented by the dot-dashed line in fig. 7

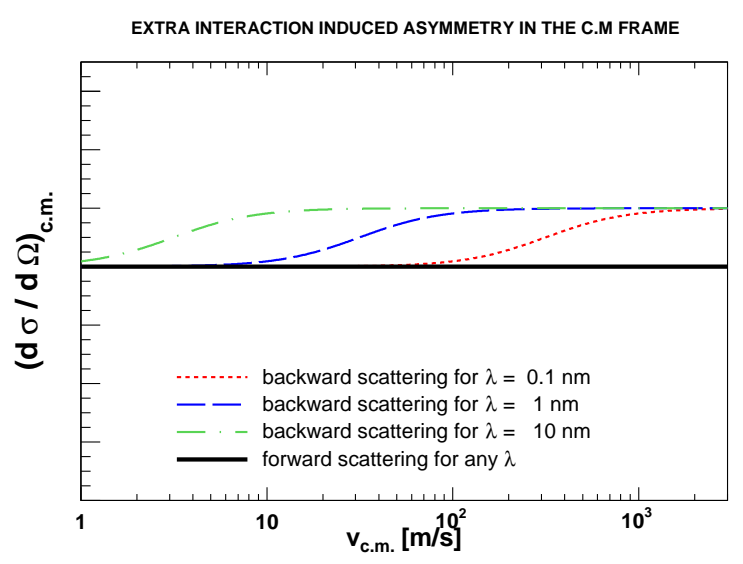

FIG. 5: The asymmetry of neutron scattering at an atom in the center-of-mass reference system is shown as a function of various characteristic ranges of the additional interaction 0,$1 ; 1 ; 10 \mathrm{~nm}$.

\section{ASYMMETRY OF SCATTERING}

As is clear from fig. 7 , the best constraint was obtained from the analysis of the energy dependence of the neutron scattering lengths in the $b_{n e}$ measurements. However, the precision here is limited by the correction for the $b_{n e}$ value itself. An obvious proposal for improving this constraint would be to set up experimental conditions free of the $b_{n e}$ contribution. This is indeed possible, because neutron-electron scattering is essential for fast neutrons only, and is absent for slow neutrons.

We propose improving the experiment 24 and measuring the forward-backward asymmetry of the scattering of neutrons at atoms of noble gases, in the following way: the initial velocity of the neutrons should correspond to the range of very cold neutrons ( $\mathrm{VCN})$; the double differential measurement of neutron velocity before/after scattering should be used to calculate the transferred momentum for every collision.

Fig. [5] shows the asymmetry of neutron scattering at an atom in the center-of-mass reference system, as a function of various characteristic ranges of the additional interaction. It is clear that neutrons with velocities of $1-1000 \mathrm{~m} / \mathrm{s}$ need to be used. This means that the thermal motion of atoms cannot be neglected, and that the neutron velocity in the laboratory fixed reference system is not equal to that in the center-of-mass reference system. Even totally isotropic scattering in the centerof-mass reference system would be highly anisotropic in the laboratory system. Nevertheless, the kinematics of the scattering process can be reconstructed precisely if both the initial and the final neutron velocity in the laboratory reference system are measured. This can easily be done with reasonable statistical accuracy for thermal, cold, very cold and probably even for ultracold neutrons. In such a case, the asymmetry calculated would be modified by an additional short-range interaction as shown in 

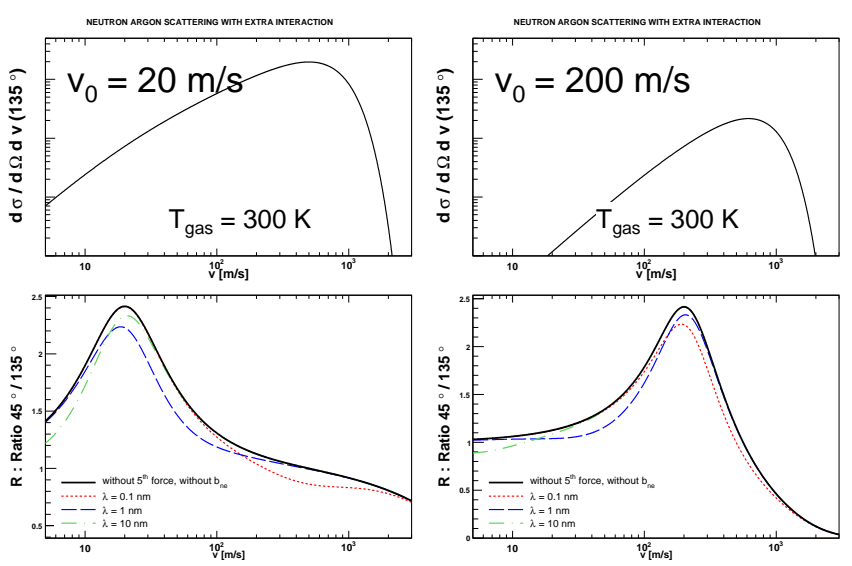

FIG. 6: The bottom two graphs show the ratio of neutron flux scattered at an argon atom at an angle of 45 degrees (forward) to that scattered at an angle of 135 degree (backward), as a function of the final neutron velocity for the following cases: no additional interactions, additional interactions with characteristic ranges of $0.1 ; 1 ; 10 \mathrm{~nm}$. The thermal motion of gas is taken into account. The top two graphs indicate the rate of collision with argon atoms (in thermal motion) of neutrons with a fixed initial neutron velocity. The initial neutron velocity for the two graphs on the left is equal to $20 \mathrm{~m} / \mathrm{s}$; the initial neutron velocity for the two graphs on the right is equal to $200 \mathrm{~m} / \mathrm{s}$.

fig. 66 (the thermal motion of argon atoms is taken into account).

The measurement described above could easily provide an accuracy of $10^{-3}$ for the ratio of forward to backward scattering probabilities and a corresponding constraint for the additional short-range interactions shown in fig. 7 . The relative drop in sensitivity at a few times $10^{-11} \mathrm{~m}$ is due to the appearance of neutron electron scattering; the range of interest for this possible constraint is $10^{-11}$ $10^{-8} \mathrm{~m}$.

\section{CONCLUSION}

We analysed the constraints for extra short-range interactions on the basis of the existing data on neutron scattering. These constraints are several orders of magnitude better than those usually cited in the range between $1 \mathrm{pm}$ and $5 \mathrm{~nm}$. The reliability of these constraints was supported by the application of several independant methods with comparable accuracy, as well as by the use of a major fraction of known neutron scattering lengths and treatment of the data in a most conservative way. One constraint obtained within the random potential nuclear model was based on the absence of an additional linear term in the mass dependance of the neutron scattering lengths. It would be difficult to improve this constraint in either experimental or theoretical terms. Another constraint was derived by comparing two

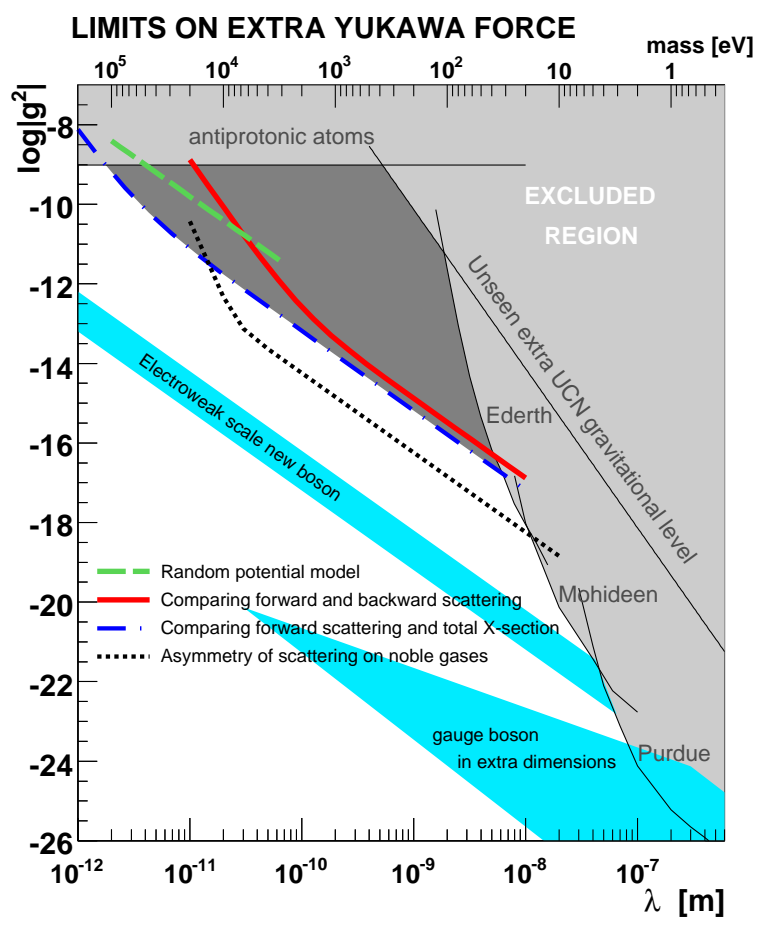

FIG. 7: Constraint on extra Yukawa force at $95 \%$ C.L. (dashed, dot-dashed and bold lines) obtained in this article. The dotted line is an estimation of the sensitivity of the proposed experiment. Also shown the existing contraints [11, 13] and the theoretical regions of interest [4, 10].

types of neutron scattering experiments with different sensitivities to the extra short-range interactions. These are interference experiments measuring forward neutron scattering and the Bragg diffraction. The accuracy here is limited by the relatively poor precision of the Bragg scattering technique. Significant improvements in the accuracy of such experiments would be particularly interesting. Further constraints were estimated using the energy-dependence of the neutron scattering lengths at heavy nuclei. They are limited by the precision of our knowledge of the neutron-electron scattering length. An elegant method for further improving such constraints would consist in achieving experimental conditions free of $b_{n e}$ contribution. This is indeed possible, given that neutron-electron scattering is essential for fast neutrons only. The experiment would consist in scattering very cold neutrons at rare noble gases and in measuring precisely the differential asymmetry of such scattering as a function of the transferred momentum.

\section{Acknowledgement}

We are grateful for a number of stimulating discussions with our colleagues from the GRANIT project and the participants of the GRANIT 2006 workshop. This 
work is supported by the French Agence Nationale de la Recherche (ANR).

[1] W.-M. Yao et al., J. Phys. G 33, 1 (2006).

[2] T. D. Lee and C. N. Yang, Phys. Rev. 98, 1501 (1955).

[3] D. J. Kapner et al., Phys. Rev. Lett. 98, 021101 (2007).

[4] N. Arkani-Hamed, S. Dimopoulos and G. R. Dvali, Phys. Lett. B 429, 263 (1998); Phys. Rev. D 59, 086004 (1999).

[5] I. Antoniadis et al., Phys. Lett. B 436, 257 (1998).

[6] V. A. Rubakov and M. E. Shaposhnikov, Phys. Lett. B 125, 136 (1983); Phys. Lett. B 125, 139 (1983).

[7] M. Visser, Phys. Lett. B 159, 22 (1985).

[8] I. Antoniadis, Phys. Lett. B 246, 377 (1990).

[9] J. D. Lykken, Phys. Rev. D 54, 3693 (1996).

[10] P. Fayet, Phys. Rev. D 75115017 (2007); P. Fayet, arXiv:hep-ph/0111282.

[11] M. Bordag, U. Mohideen and V. M. Mostepanenko, Phys. Rept. 353, 1 (2001).

[12] M. Hori et al., Phys. Rev. Lett. 91, 123401 (2003).

[13] V. V. Nesvizhevsky and K. V. Protasov, Class. Quant. Grav. 21, 4557 (2004).

[14] H. Leeb and J. Schmiedmayer, Phys. Rev. Lett. 68, 1472 (1992).

[15] A. Frank, P. van Isacker and J. Gomez-Camacho, Phys. Lett. B 582, 15 (2004).

[16] P. J. S. Watson, arXiv:hep-ph/0406308

[17] G. L. Greene and V. Gudkov, Phys. Rev. C 75, 015501 (2007).
[18] V. V. Nesvizhevsky, G. Pignol and K. V. Protasov, Proceedings of the 42th Rencontres de Moriond (2007).

[19] S. Baeßler, V. V. Nesvizhevsky, K. V. Protasov and A. Y. Voronin, Phys. Rev. D 75, 075006 (2007).

[20] V. V. Nesvizhevsky et al., Nature 415297 (2002).

[21] V.F. Sears, Phys. Reports 141, 281 (1986).

[22] E. Fermi, L. Marshall, Phys. Rev. 71, 666 (1947).

[23] L. Koester, H. Rauch and E. Seymann, Atomic Data and Nuclear Data Tables 49, 65 (1991).

[24] V. E. Krohn and G. R. Ringo, Phys. Rev. 148, 1303 (1966).

[25] M. Peskhin and G. R. Ringo, Am. J. Phys. 39, 324 (1971).

[26] S. Kopecky, J. A. Harvey, N. W. Hill, M. Krenn, M. Pernicka, P. Riehs, and S. Steiner, Phys. Rev. C56, 2229 (1997).

[27] S. Glaster et al., Nucl. Phys. B 32, 221 (1971);

A. F. Krutov and V. E. Troitsky, Eur. Phys. J. A 16, 285 (2003).

[28] http://www.jlab.org/ cseely/formfactor_data.txt

[29] For simplicity, the amplitude due to electron-neutron scattering has been omitted for the time being. This term will be given detailed treatment in section $\mathrm{V}$ 